\title{
O uso da eletroconvulsoterapia (ECT) para o tratamento da depressão
}

The use of electroconvulsive therapy (ECT) for the treatment of depression

El uso de la terapia electroconvulsiva (TEC) para el tratamiento de la depresión

Isabelle Salomão Teixeira Silva ${ }^{1 *}$, Jhonson Tizzo Godoy², Ana Paula Gouvêa de Barros ${ }^{3}$, luri Carvalho Vieira ${ }^{4}$, Isabella de Moura Magalhães ${ }^{5}$, Manuela Pittella de Mattos ${ }^{6}$, Raquel Carmo Araújo ${ }^{7}$, Victório de Moura Magalhães ${ }^{8}$, Adriel Gustavo Lopes ${ }^{1}$.

\section{RESUMO}

Objetivo: Avaliar a eficácia da eletroconvulsoterapia (ECT) no tratamento da depressão, considerando seus benefícios e comparando sua eficácia entre depressão unipolar e bipolar. Além de também observar o risco de surgimento de episódios de mania após o uso da ECT. Revisão Bibliográfica: Atualmente, embora seja uma terapêutica muito promissora, o uso da ECT tem sido reservado para casos refratários ao tratamento medicamentoso, em função de ainda ser uma ferramenta pouco disponível para uso na prática clínica. De acordo com as evidências atuais quanto menor o tempo de duração da doença, maiores são as taxas de resposta e remissão dos sintomas com o uso da terapia eletroconvulsiva, sendo que alguns estudos afirmam que a taxa de resposta da depressão bipolar é maior que da depressão unipolar. Alguns efeitos adversos foram percebidos, entre eles o desenvolvimento de episódios maníacos em pacientes previamente deprimidos. Considerações finais: Os estudos demonstraram melhora clínica dos pacientes com transtorno depressivo bipolar e unipolar utilizando a ECT como tratamento, em especial na depressão bipolar. Foi observado a ocorrência de episódios de mania após o uso dessa terapêutica, como um efeito adverso. No entanto, faz-se necessário mais pesquisas no intuito de diminuir o estigma e obter mais dados.

Palavras-chave: Depressão, Eletroconvulsoterapia, Transtorno bipolar.

\begin{abstract}
Objective: To evaluate the effectiveness of electroconvulsive therapy (ECT) in the treatment of depression, considering its benefits and comparing its effectiveness between unipolar and bipolar depression. In addition, to observe the risk of episodes of mania after the use of ECT. Bibliographic Review: Currently, although it is a very promising therapy, the use of ECT has been reserved for cases refractory to drug treatment, because it is still a tool that is not widespread in clinical practice. According to current evidence, the shorter the duration of the disease, the higher the response and remission rates of symptoms with the use of electroconvulsive therapy, with some studies claiming that the response rate of bipolar depression is higher than that of depression unipolar. Some adverse effects were noticed, including the development of manic episodes in previously depressed patients. Final considerations: Studies have shown clinical improvement in patients with bipolar and unipolar depressive disorder using ECT as a treatment, especially in bipolar depression. It was observed the occurrence of manic episodes after the use of this therapy, as an adverse effect. However, more research is needed to reduce stigma and obtain more data.
\end{abstract}

Key words: Depression, Electroconvulsive therapy, Bipolar disorder.

1 Pontifícia Universidade Católica de MG (PUC-MG), Betim - MG. *E-mail: isabellesilva554@gmail.com

2 Santa Casa de Misericórdia de Belo Horizonte, Belo Horizonte - MG.

${ }^{3}$ Universidade Nove de Julho (UNINOVE), São Paulo - SP.

${ }^{4}$ Centro Universitário Governador Ozanam Coelho (UNIFAGOC), Ubá - MG.

${ }^{5}$ Centro Universitário Presidente Tancredo de Almeida Neves (UNIPTAN), São João Del Rei - MG.

${ }^{6}$ Faculdade de Saúde e Ecologia Humana (FASEH), Belo Horizonte - MG.

${ }^{7}$ Centro Universitário de Belo Horizonte (UNIBH), Belo Horizonte - MG.

${ }^{8}$ Faculdade de Ciências Médicas e da Saúde de Juiz de Fora (SUPREMA), Juiz de Fora - MG. 


\section{RESUMEN}

Objetivo: Evaluar la efectividad de la terapia electroconvulsiva (ECT) em el tratamiento de la depresión, considerando sus beneficios y comparando suefectividad entre la depresión unipolar y bipolar. Además de observar también el riesgo de episodios de manía después del uso de ECT. Revisión bibliográfica: Actualmente, aunque es una terapia muy prometedora, el uso de ECT se ha reservado para casos refractarios al tratamiento farmacológico, porque todavía es una herramienta que no está muy extendida em la práctica clínica. Según la evidencia actual, cuanto menor es la duración de la enfermedad, mayores son lastasas de respuesta y remisión de los sintomas com el uso de la terapia electroconvulsiva, y algunos estúdios afirman que latasa de respuesta de la depresión bipolar es mayor que la de la depresión. Unipolar. Se notaron algunos efectos adversos, incluído el desarrollo de episodios maníacos en pacientes previamente deprimidos. Consideraciones finales: Los estudios han demostrado una mejoría clínica en pacientes con trastorno depresivo bipolar y unipolar que utilizan la TEC como tratamiento, especialmente en la depresión bipolar. Se observó la aparición de episodios maníacos después del uso de esta terapia, como un efecto adverso. Sin embargo, se necesita más investigación para reducir el estigma y obtener más datos.

Palabras clave: Depresión, Terapia electroconvulsiva, Trastorno bipolar.

\section{INTRODUÇÃO}

Atualmente, o Manual de Diagnóstico e Estatístico de Transtornos Mentais - $5^{a}$ edição (DSM 5) apresenta diversos tipos de classes diagnósticas relacionadas a psiquiatria. Dentre elas, encontra-se o Transtorno Bipolar e outros transtornos relacionados, no qual está inserido o Transtorno Bipolar tipo 1 e tipo 2, e os transtornos depressivos, que englobam o transtorno depressivo maior (RIBEIRO AS, et al., 2020).

O Transtorno Bipolar (TB) é uma doença crônica, prevalente, recorrente e debilitante, que se caracteriza por alterações na afetividade, com episódios de mania e depressão. Esse transtorno está dividido em 2 tipos: o tipo 1, que requer a ocorrência de pelo menos um episódio maníaco e o tipo 2, que é caracterizado por pelo menos um episódio hipomaníaco e um depressivo maior (RODRIGUES PMS, 2017). Esses episódios depressivos em indivíduos com Transtorno Bipolar também podem ser conhecidos como depressão bipolar.

Pode-se mencionar também que esse transtorno afeta homens e mulheres de maneira diferentes, sendo as taxas de prevalência do TB do tipo I maiores nos homens, enquanto as taxas do TB do tipo II são maiores nas mulheres.

Outro distúrbio psiquiátrico que está presente em grande parte da população mundial é o Transtorno Depressivo Maior (TDM), conhecido também somente como depressão ou como depressão unipolar. Essa doença que pode trazer prejuízos laborais e sociais e tem como característica a presença de episódios depressivos sem a presença do humor maníaco (BEZERRA LFD, 2018).

Os episódios de depressão, tanto na depressão bipolar que ocorre no TB, como na depressão unipolar ou TDM, caracterizam-se por várias alterações no aspecto afetivo do paciente que consiste no sentimento de melancolia, tristeza, baixa autoestima e muitas vezes leva o paciente a perder o interesse pela vida. $O$ paciente encontra-se com uma postura cabisbaixa como olhar baixo, falta de higiene e interesse, podendo ainda haver ainda alterações somáticas como sonolência.

Já os episódios de mania, são manifestações contrárias a depressão, nela ocorre a exaltação dos sentimentos, ou seja, o humor expandido, nesses casos, o paciente encontra-se com a autoestima elevada, muitas vezes extravagante, podendo inclusive aparecer como delírios de grandeza. As alterações somáticas também se opõem às da depressão, por exemplo, a depressão pode apresentar-se com sonolência, já no caso da mania, é comum ocorrer insônia (BEZERRA LFD, 2018; RODRIGUES PMS, 2017).

Atualmente, há diversos tipos de tratamentos para esses tipos de doenças psiquiátricas, dentre eles, incluise a eletroconvulsoterapia (ECT) que é um procedimento de estimulação cerebral que apresenta alta eficácia (DJ L, et al., 2015; PINNA M, et al., 2018). 
Este procedimento surgiu no Brasil em meados do século 20 e foi desenvolvida em hospitais psiquiátricos. Contudo, no final do século 20, com o surgimento dos psicofármacos e com movimentos antagônicos ao seu uso, a prática entrou em decadência (GUIMARÃES NA, et al., 2013; MIRANDA JS, et al., 2019).

A técnica da eletroconvulsoterapia foi utilizada pela primeira vez por Cerlleti e Bini em 1939 para induzir um estado convulsivo em um paciente que apresentava sintomas psicóticos. Após a primeira sessão observou-se melhora com total remissão após 11 sessões de ECT (MEDDA P, et al., 2014). Anteriormente, a essência da técnica já havia sido adotada por Ladhaus Von Meduna em 1885, que descreveu benefícios da convulsão induzida por cânfora, que melhorava os sintomas psiquiátricos após a indução de convulsões. Outra terapia utilizada era o uso de insulina. A popularidade da técnica da eletroconvulsoterapia, contudo, entrou em declínio na década de 60 , em parte pela introdução de classes de psicofármacos e a luta antimanicomial (MOSER CM, et al., 2015).

Desde então, a ECT foi sendo utilizada como a terapia física mais comum para transtornos de humor, como episódios depressivos, mistos ou maníacos de transtorno bipolar ou transtorno depressivo maior. Por suas características terapêuticas, a ECT pode ser considerada o primeiro tratamento que apresenta propriedades estabilizadoras de humor sendo iniciada 10 anos antes do descobrimento do lítio. Entende-se como um estabilizador de humor "um agente que demonstra igual eficácia no tratamento agudo de mania ou depressão como na prevenção de episódios de transtorno de humor (definição ideal) ou um agente que demonstra eficácia em um desses aspectos" (MEDDA P, et al., 2014).

Há indicações atuais para a eletroconvulsoterapia, uma vez que ela foi aperfeiçoada e padronizada para a redução de efeitos adversos, como fraturas, deslocamento de membros e declínios cognitivos importantes. Dentre os aperfeiçoamentos adotados, estão o uso de relaxantes musculares e anestésicos, além da padronização da carga elétrica e o posicionamento de eletrodos. Porém, mesmo com a existência de indicações atuais, há resistência em relação à adoção da eletroconvulsoterapia, principalmente pela estigmatização relacionada ao seu uso histórico e interesse maior em desenvolvimento e disseminação do uso de psicofármacos pela indústria farmacêutica (MOSER CM, et al., 2015).

Segundo o estudo de Bahji A, et al. (2019) apenas 1\% dos pacientes com depressão refratária e 0,25\% dos pacientes com transtorno bipolar e unipolar nos EUA já utilizaram ECT nos EUA, o que demonstra uma subutilização da técnica, apesar desta ser indicada em caso de falha terapêutica farmacológica ou severidade dos sintomas como risco de suicídio, sintomas psicóticos, catatonia ou inapetência levando à desnutrição (DJ L, et al., 2015).

Acerca do mecanismo da ECT para o tratamento desse transtornos, ainda não se sabe ao certo se ela atua provocando um reajuste nos receptores de serotonina ou se aumenta o limiar convulsivo atuando como um estabilizador de humor ou se aumenta o metabolismo e o fluxo sanguíneo em certas áreas cerebrais através de uma teoria pouco elucidada sobre a depressão. Sendo que o aumento do limiar convulsivo também é considerado como uma hipótese (DJ L, et al., 2015).

Portanto, o objetivo deste artigo é avaliar a eficácia da ECT no tratamento da depressão, considerando seus benefícios e comparando sua eficácia entre depressão unipolar e bipolar. Além de também observar o risco de surgimento de episódios de mania após o uso da ECT.

\section{REVISÃO BIBLIOGRÁFICA}

O uso da eletroconvulsoterapia (ECT), embora ainda estigmatizado entre muitos médicos, tem demonstrado eficácia, segurança e estabilidade no tratamento da depressão, em especial no Transtorno Bipolar (MOSER CM, et al., 2015). Além disso, a ECT é estatisticamente mais eficaz e requer menor número de sessões em pacientes com depressão bipolar quando comparados com pacientes com depressão unipolar (AGARKAR S, et al., 2018; BAHJI A, et al., 2019; PINNA M, et al., 2018).

Em contrapartida, o estudo de Medda $\mathrm{P}$, et al. (2014) defende que a ECT demonstra similar eficácia tanto na depressão bipolar e unipolar, embora reconheça que há poucos estudos disponíveis tratando-se da depressão bipolar. 
Este mesmo artigo cita como fonte um estudo clínico prospectivo que alega a polaridade não ser um fator preditivo de taxa de resposta à ECT, sendo que a taxa de resposta encontrada foi de aproximadamente $60 \%$ para ambos os transtornos.

Ademais, com a ECT tem-se obtido melhores resultados em relação a sintomatologia dos pacientes com depressão refratária quando comparado aos resultados obtidos dos mesmos com o tratamento farmacológico. Para tal fato, pode-se mencionar um estudo com 3 casos conduzidos à ECT devido à baixa resposta por tratamentos medicamentosos antipsicóticos e parenterais. Dos 3 pacientes submetidos a ECT, 2 apresentaram resultados positivos com resolução completa da sintomatologia. Já o terceiro caso, obteve resposta parcial e necessitou de um outro subtipo de estimulação magnética. Desse estudo, nenhum paciente apresentou alteração cognitiva ou outros efeitos colaterais (SIDROV A, et al., 2016). Tal achado, contrariou alguns autores que defendem a ocasião de efeitos colaterais através da ECT (DJ L, et al., 2015).

Além dos estudos anteriores terem demonstrado uma melhora significativa ou total da sintomatologia dos pacientes com depressão com o uso da ECT, foi também encontrado que a terapia eletroconvulsiva tem as maiores taxas de resposta e remissão do que qualquer forma de tratamento antidepressivo. Tal dado é frequente entre pacientes com transtorno depressivo maior ou unipolar quando não há resposta a psicoterapêuticos e/ou intervenções farmacológicas. Bem como, quando uma resposta antidepressiva particularmente rápida é necessária, no caso de pacientes gravemente doentes (DJ L, et al., 2015).

Além disso, outro estudo demonstra que tanto os pacientes com transtorno bipolar, quanto os pacientes que apresentavam transtorno depressivo maior ou unipolar, ao serem submetidos à ECT obtiveram melhora clínica dos seus sintomas. Nessa revisão sistemática, 4 (quatro) estudos mostraram maior velocidade de resposta na depressão bipolar e 1 (um) demonstrou tendência mais rápida de erradicação de episódio depressivo no transtorno bipolar.

Desses estudos, 3 (três) dos 4 (quatro) que relataram resposta mais rápida eram prospectivos e o estudo que relatou menor tempo de internação na depressão bipolar era retrospectivo. Nenhum estudo mostrou resposta mais rápida nos pacientes com depressão maior (AGARKAR S, et al., 2018). Por outro lado, Medda $\mathrm{P}$, et al. (2014) observaram que significativamente menos pacientes com transtorno bipolar tipo I (35\%) ou tipo II (43\%) remeteram em comparação com pacientes com Transtorno Depressivo Maior (71\%).

Tais diferenças nas velocidades das respostas nos pacientes com transtorno bipolar e depressivo maior não foram atribuídas por diferenças em colocação de eletrodos. Entretanto, diversas características clínicas foram examinadas, tais como a idade em que os sintomas se manifestaram, o número de episódios, a duração dos episódios e os subtipos de bipolaridade, sendo que nenhuma dessas características refletiram influência na velocidade de resposta (DALY JJ, et al., 2001; SACKEIM HA, et al., 2005; SIENAERT P, et al., 2009).

O estudo de Daly JJ, et al. (2001), sugere que um aumento acumulativo do limiar convulsivo durante a ECT pode subestimar a resposta mais rápida dos sintomas em um diagnóstico bipolar comparado ao transtorno depressivo maior. Este estudo apresenta como suporte à esta hipótese uma tendência à um aumento maior do limiar convulsivo observado no transtorno bipolar comparado ao transtorno depressivo maior. As limitações incluem o número pequeno de estudos incluídos para tal discussão.

O estudo de Bahji A, et al. (2019) comprova que a ECT utilizada no transtorno bipolar possui alta eficácia quando comparada ao transtorno depressivo maior, sendo necessário um menor número de sessões em episódios depressivos sob o diagnóstico de transtorno bipolar, ou seja, apesar das taxas de remissão serem equivalentes $(52,3 \%)$ entre os dois quadros de depressão, a taxa de resposta da depressão bipolar foi maior $(77,1 \%)$ que da depressão maior $(74,2 \%)$, uma vez que, a primeira necessitou de um menor número de sessões. Para os autores desse estudo a diferença nas taxas de respostas foram atribuídas à heterogeneidade dos estudos e consideradas pouco relevantes na prática clínica.

Todos os pacientes analisados nos estudos abordados nessa revisão sistemática foram reportados como tendo um número similar de episódios prévios e duração do episódio atual, sem diferenças significativas na intensidade dos sintomas. Todos foram submetidos ao tratamento farmacológico, conforme os protocolos e foram incluídos como pacientes refratários à terapêutica inicial. Complementando os aspectos abordados acima, uma metanálise aborda que a presença de diagnóstico bipolar não foi um preditor significativo de 
melhor resposta à ECT, juntamente com outras variáveis - sexo, idade de início e número de episódios anteriores (HAQ AU, et al., 2015).

O estudo de Narayanaswamy JC, et al. (2014), conduzido em um ambiente hospitalar com 44 pacientes diagnosticados com transtorno bipolar e 106 pacientes com depressão unipolar refratária, também corrobora com essa ideia e observou que não houve diferença significativa entre os dois grupos em relação à eficácia da ECT, número de sessões necessárias e tempo de internação, além da velocidade de resposta e tempo de remissão.

Entretanto, a idade de surgimento dos sintomas, o uso concomitante de anticonvulsivantes e a severidade da doença foram estatisticamente significantes entre os grupos e pacientes com diagnóstico de depressão unipolar que apresentaram maior grau de severidade do transtorno e ideação suicida comparado ao grupo diagnosticado com depressão bipolar, que apresentou menor idade do início dos sintomas.

Então a maioria dos artigos analisados conseguiu relatar resposta à ECT tanto no transtorno bipolar como em episódios depressivos, sendo utilizada após falha medicamentosa como terapia de segunda linha e embora tenha-se notado uma certa resistência ao seu uso e estigmatização.

A maioria dos autores citam a necessidade de mais estudos que possam avaliar biomarcadores clínicos confiáveis para o papel da ECT, dentro de um protocolo que seja bem estabelecido quanto à predição de sua taxa de resposta à diversas variáveis que influenciam na depressão e no transtorno bipolar (HAQ AU, et al., 2015; AGARKAR S, et al., 2018; BAHJI A, et al., 2019).

Estudos recentes afirmam que pelo menos $5 \%$ da população geral já apresentou mania ou hipomania (MORENO RA, et al., 2005). De acordo com o livro texto Paulo Dalgalarrondo (2008), a mania franca é a forma mais intensa da mania, com taquipsiquismo acentuado, agitação psicomotora importante, heteroagressividade, fuga de ideais e delírio de grandeza. Ademais, ele define hipomania como uma forma atenuada de episódio maníaco, o que muitas vezes pode passar despercebido ao diagnóstico. De acordo com as manifestações clínicas do paciente, este pode apresentar transtorno afetivo bipolar do tipo I ou II.

Conforme o artigo trazido na revista da USP, o paciente que apresenta transtorno afetivo bipolar do tipo I terá episódios de mania, já o paciente do Tipo II, em que a elevação do humor é mais branda e breve, demonstrará episódios de hipomania. Nos dois casos, seja manifestando episódios de mania ou hipomania, há ciclagem com episódios depressivos (BOISAPO N, et al. 2017).

Ao falar sobre a epidemiologia do transtorno bipolar (TB), dados mundiais demonstram que esse transtorno afeta homens e mulheres de maneira diferentes. Notou-se que as taxas de prevalência do TB do tipo I foram maiores nos homens enquanto as mulheres apresentaram taxas mais elevadas do TB do tipo II. Ainda sobre a epidemiologia da TB, um outro estudo analisado também evidenciou que o risco de se desenvolver o TB é maior em adultos jovens e que pelo menos metade dos casos se iniciam antes dos 25 anos (BOISAPO N, et al., 2017).

Um fato interessante é que metade dos pacientes com TB apresentam no início da doença um episódio de mania, entretanto a outra metade apresenta sintomas depressivos, podendo receber erroneamente 0 diagnóstico de depressão unipolar (BOISAPO N, et al., 2017). Em outro artigo, é elucidado o fato da possibilidade de uma pessoa ser diagnosticada e receber tratamento para transtorno depressivo unipolar e posteriormente, com o aparecimento de sintomas maníacos, concluir-se que se tratava de um quadro de transtorno bipolar (LI J, et al., 2015).

O mesmo artigo citado acima, traz o relato de caso de uma paciente: sexo feminino, 40 anos, que apresenta episódios depressivos maiores repetidamente até os 38 anos. Desde o seu diagnóstico recebeu tratamento com alguns psicofármacos, porém persistia com ideação suicida e mau humor. Em certo momento, ela parou com todos os agentes psicotrópicos e iniciou a terapia eletroconvulsiva. Após 2 sessões, os sintomas depressivos diminuíram rapidamente e a paciente começou a manifestar sintomas de euforia e grandiosidade. Ao final da terceira sessão, a paciente tinha desenvolvido episódio maníaco. Apesar disso, a paciente continuou com a terapia eletroconvulsiva sendo que, seus sintomas de euforia e grandiosidade 
agravaram-se acentuadamente. Após 5 sessões, a ECT foi descontinuada e a paciente iniciou tratamento farmacológico, com novas medicações. Ao final de todo esse tratamento, considerando o uso da ECT e terapia farmacológica, ela obteve remissão completa dos sintomas (LI J, et al., 2015).

Entretanto, outro estudo recente apoia a eficácia da terapia eletroconvulsiva para transtorno bipolar, porque essa possui ação na manutenção do bem estar do paciente. Defendendo que a ECT moderna é adequadamente tolerada e sua taxa de conversão em mania é baixa, se as recomendações técnicas atuais e de monitoramento forem seguidas minuciosamente (AGARKAR S, et al., 2018).

Outro estudo traz dados que reportaram uma taxa de $6,4 \%$ de mania em 94 pacientes que foram admitidos com sintomas depressivos e uma taxa de $12,5 \%$ de episódios maníacos em pacientes admitidos com depressão maior. Há uma observação de que o episódio maníaco ocorre predominantemente em 24 horas após o uso da ECT e pode desaparecer gradualmente sem necessariamente haver intervenção.

Relata também que sexo, número de episódios maníacos prévios, idade ou diagnóstico de transtorno bipolar I ou II afetaram o risco de se desenvolver um episódio maníaco após ECT. O uso prévio de uma droga anticonvulsivante ou estabilizadora de humor não afetaram na manifestação de episódios maníacos. Porém história de uso prévio de lítio parece diminuir a frequência desses episódios. Tal estudo encontra que o risco de ciclagem após a ECT é baixo, principalmente comparado à pacientes com transtorno bipolar em um episódio depressivo (MEDDA P, et al., 2014).

Apesar da insuficiência de explicações, é notável que o efeito adverso da ECT seja o desenvolvimento desses episódios maníacos (DJ L, et al., 2015; BAHJI A, et al., 2019). Porém, ainda é necessário maior número de estudos e pesquisas sobre o assunto, já que por meio dos estudos lidos também foi afirmado que o sucesso da ECT e a baixa taxa de conversão em mania depende das recomendações técnicas e de monitoramento (AGARKAR S, et al., 2018).

Considerando os aspectos já mencionados, pode-se concluir que a depressão bipolar é considerada um transtorno psiquiátrico agregado a incapacidade funcional, suicídio e aumento da mortalidade. O que torna essencial a busca pelo conhecimento da terapia eletroconvulsiva como uma outra possibilidade, além da farmacoterapia, para tratamentos que não são beneficiados pelos tratamentos farmacológicos tradicionais, promovendo assim uma melhora desse transtorno e também outros benefícios para a saúde pública como a redução de gastos, a diminuição da mortalidade e a manutenção do bem estar do indivíduo (AGARKAR S, et. al., 2018).

É alto o número de pacientes com depressão não responsivo ao tratamento por terapia eletroconvulsiva, por isso conhecer preditores clínicos que beneficiam o paciente pode selecionar de forma específica aqueles que teriam melhores resultados através da ECT.

Por meio de uma meta-análise de dados foi conclusivo que quanto maior o tempo de duração dos episódios depressivos associado à uma falha terapêutica medicamentosa de base da doença, maior também é a falha relacionada a terapia eletroconvulsiva. Dados como idade e psicose não fizeram diferença para o estudo.

Portanto, é possível observar que a eficácia da ECT está atrelada a fatores como a duração dos episódios depressivos e a falha terapêutica farmacológica, sendo que quanto maior a duração desses episódios e maior a falha medicamentosa, menor seria a eficácia da ECT (HAQ AU, et al., 2015).

Portanto, é indiscutível que a ECT moderna é tolerável e possui mínimos efeitos colaterais cognitivos. Entretanto, é necessário que mais estudos sejam realizados de maneira a disponibilizar mais fatores preditivos clínicos para o tratamento de depressão utilizando a ECT (AGARKAR S, et al., 2018).

Sendo assim, por meio desses critérios seria possível melhorar e especificar o diagnóstico do paciente, além de promover a confirmação futura da excelência do tratamento individualizado para cada paciente sem gerar excesso de gastos ou outros transtornos (HAQ AU, et al., 2015). 


\section{CONSIDERAÇÕES FINAIS}

O uso da ECT embora ainda estigmatizado, vem crescendo, uma vez que foi demonstrado sua eficácia e segurança no tratamento da depressão, em especial da depressão bipolar. A maioria dos artigos analisados, conseguiu relatar resposta à ECT tanto nos episódios depressivos do transtorno bipolar como em episódios depressivos unipolares, sendo geralmente utilizada após falha medicamentosa como terapia de segunda linha. Além disso, evidenciou-se que o tratamento com a ECT em uso concomitante a uma terapia farmacológica mostrou-se mais eficaz. Outro ponto positivo do uso desse tratamento é a relação custo benefício do ECT estabelecida no caso de depressão refratária ao tratamento. Todavia, há relatos de ocorrência de episódios de mania após o uso dessa terapêutica, como um efeito adverso.

\section{REFERÊNCIAS}

1. AGARKAR S, et al. Speed of antidepressant response to electroconvulsive therapy in bipolar disorder vs. major depressive disorder. PsychiatryResearch, 2018; 265:355-359.

2. BAHJI A, et al. ECT beyond unipolar major depression: systematic review and meta-analysis of electroconvulsive therapy in bipolar depression. Acta PsychiatricaScandinavica, 2019; 139(3):214-226.

3. BEZERRA LFD. Associação entre transtorno depressivo maior e qualidade de vida em domiciliados de um bairro vulnerável de uma capital do Nordeste. Dissertação (Mestrado em Enfermagem) - Escola de Enfermagem e Farmácia, Programa de Pós-Graduação em Enfermagem. Universidade Federal de Alagoas, Maceió, 2018; $112 p$.

4. BOSAIPO N, et al. Transtorno bipolar: uma revisão dos aspectos conceituais e clínicos. Medicina (Ribeirão Preto), 2017; 50(1):72-84.

5. DALGALARRONDO P. Psicopatologia e semiologia dos transtornos mentais [recurso eletrônico]. 2. ed. Porto Alegre: Artmed, 2008; 438p.

6. DALY JJ, et al. ECT in bipolar and unipolar depression: differences in speed of response. Bipolar Disorders, 2001; 3(2):95-104.

7. DJ L, et al. Electroconvulsive Therapy-Induced Manic Episode for a Patient With Bipolar Depression A Case Report. Journalof ECT, 2015; 31:31-31.

8. GUIMARÃES NA, et al. Tratamento em saúde mental no modelo manicomial (1960 a 2000): histórias narradas por profissionais de enfermagem. Texto contexto - enferm., 2013; 22(2):361-369.

9. HAQ AU, et al. Response of Depression to Electroconvulsive Therapy: A Meta-Analysis of Clinical Predictors. The journal of clinical psychiatric, 2015; 76(10):1374-1384.

10. IRAURGI I, et al. Assessment of individual clinical outcomes: Regarding an electroconvulsive therapy. Revista de Psiquiatría y Salud Mental (EnglishEdition), 2015; 8(1):11-16.

11. LI J, et al. Electroconvulsive Therapy-Induced Manic Episode for a Patient With Bipolar Depression: a case report. Journal of ECT, 2015; 31(2).

12. MEDDA P, et al. The Mood-Stabilizing Effects of Electroconvulsive Therapy. Journalof ECT, 2014; 30(4):275-282.

13. MIRANDA JS, et al. Convulsoterapias na prática psiquiátrica brasileira. Rev. Enf. Ref. [online], 2019; 4(21):159-168.

14. MORENO RA, et al. Diagnóstico, tratamento e prevenção da mania e da hipomania no transtorno bipolar. Rev. psiquiatr. clín., 2005; 32(1):39-48.

15. MOSER CM, et al. Evidências da eficácia da eletroconvulsoterapia na prática psiquiátrica. Rev. psiquiatr., 2005; 27(3):302-310.

16. MUTZ J, et al. Comparative efficacy and acceptability of non-surgical brain stimulation for the acute treatment of major depressive episodes in adults: systematic review and network meta-analysis. BMJ, 2019; 364:1079.

17. NARAYANASWAMY JC, et al. Efficacy of ECT in bipolar and unipolar depression in a real life hospital setting. Asian Journal of Psychiatry, 2014; 8:43-46.

18. PINNA $M$, et al. Clinical and biological predictors of response to electroconvulsive therapy (ECT): a review. Neuroscience Letters, 2018; 669:32-42.

19. RIBEIRO AS, et al. Psicopatologia na contemporaneidade: análise comparativa entre o DSM-IV e o DSM-V. Fractal, Rev. Psicol., 2020; 32(1):46-56.

20. RODRIGUES PMS. Transtorno bipolar I e II: fatores sociodemográficos, comorbidades psiquiátricas, risco de suicídio e qualidade de vida. Dissertação (Mestrado em Enfermagem) - Escola de Enfermagem e Farmácia, Programa de PósGraduação em Enfermagem, Universidade Federal de Alagoas, Maceió, 2017; 177 p.

21. SACKEIM HA, et al. A prospective, randomized, double-blind comparison of bilateral and right unilateral electroconvulsive therapy at different stimulus intensities. ArchGenPsychiatry, 2000; 57(5):425-434.

22. SIDROV A, et al. Rapid amelioration of severe manic episodes with right unilateral ultrabrief pulse ECT: a case series of four patients. AustralasianPsychiatry, 2016; 10-12.

23. SIENAERT P, et al. Ultra-brief pulse ECT in bipolar and unipolar depressive disorder: differences in speed of response. Bipolar Disorders, 2009; 11(4):418-424. 